\title{
Phylogenetic relationships of closely-related phlebotomine sand flies (Diptera: Psychodidae) of Nyssomyia genus and Lutzomyia subgenus
}

\author{
Sofía Lorián Moya ${ }^{1,2}$, Angélica Pech-May ${ }^{1,2 /+}$, María Gabriela Quintana ${ }^{1,2,3}$, \\ Mariana Manteca-Acosta ${ }^{2,4}$, Oscar Daniel Salomón ${ }^{1,2}$ \\ ${ }^{1}$ Ministerio de Salud de la Nación, Administración Nacional de Laboratorios e Institutos de Salud Dr Carlos G Malbrán-ANLIS, \\ Instituto Nacional de Medicina Tropical, Puerto Iguazú, Misiones, Argentina \\ ${ }^{2}$ Consejo Nacional de Investigaciones Científicas y Técnicas, Buenos Aires, Argentina \\ ${ }^{3}$ Universidad Nacional de Tucumán, Instituto Superior de Entomología, San Miguel de Tucumán, Argentina \\ ${ }^{4}$ Ministerio de Salud de la Nación, Administración Nacional de Laboratorios e Institutos de Salud Dr Carlos G Malbrán-ANLIS, \\ Centro Nacional de Diagnóstico e Investigación en Endemo-Epidemias, Buenos Aires, Argentina
}

BACKGROUND The Nyssomyia genus and Lutzomyia subgenus include medical important species that are Latin American leishmaniases vectors. Little is known about the phylogenetic relationships of closely-related species in each of these taxonomic groups that are morphologically indistinguishable or differentiated by very subtle details.

OBJECTIVES We inferred the phylogenetic relationships of closely-related species within both the Nyssomyia genus and the Lutzomyia subgenus using a cytochrome $c$ oxidase subunit I (COI) fragment.

METHODS The sampling was carried out from 11 Argentinean localities. For genetic analyses, we used GenBank sequences in addition to our sequences from Argentina. Kimura 2-parameter (K2P) genetic distance and nucleotide divergence $(D a)$ was calculated between closely-related species of Nyssomyia genus, Lutzomyia subgenus and between clades of Lutzomyia longipalpis complex.

FINDINGS The K2P and Da values within species of Nyssomyia genus and Lutzomyia subgenus were lower than the divergence detected between clades of Lu. longipalpis complex. The haplotype network analyses within Lutzomyia subgenus showed shared haplotypes between species, contrary to Nyssomyia genus with none haplotype shared. Bayesian inference within Nyssomyia genus presented structuring by species.

MAIN CONCLUSIONS This study evidences the phylogenetic proximity among closely-related species within Nyssomyia genus and Lutzomyia subgenus. The COI sequences of Nyssomyia neivai derived from the present study are the first available in GenBank.

Key words: phlebotomine sand flies - vectors - cytochrome $c$ oxidase subunit 1 (COI)

Phlebotomine sand flies (Diptera: Psychodidae) are involved in the transmission of arboviruses, bacteria and protozoans. Among these pathogens, the trypanosomatids of the genus Leishmania spp. Ross are the etiological agents of the leishmaniases, a zoonotic disease considered as neglected with special relevance in public health. (1) Over 500 phlebotomine sand flies species distributed among 23 genera are known to occur in the Americas. (2) Inside Nyssomyia genus, Nyssomyia intermedia (Lutz \& Neiva), Nyssomyia whitmani (Antunes \& Coutinho) and Nyssomyia neivai (Pinto) are Leishmania braziliensis Vianna vectors. ${ }^{(1,3)}$ Nyssomyia whitmani is also a Leishmania shawi Lainson, de Souza, Póvoa, Ishikawa \& Silveira vector and is suspected to transmit Leishmania guyanensis Floch. ${ }^{(3,4)}$ Nyssomyia intermedia and $N y$. whitmani are widely distributed in Brazil, including the

\footnotetext{
doi: 10.1590/0074-02760200220

Financial support: Centre of Biodiversity Genomics, University of Guelph, Canada and Small Grants Programme - a joint initiative of the PAHO/TDR and the Special Programme for Research and Training in Tropical Diseases, UNICEF, UNDP, the World Bank and WHO.

SLM and AP-M contributed equally to this work.

+ Corresponding author: apechmay@gmail.com

(1) https://orcid.org/0000-0003-4942-7788

Received 08 May 2020

Accepted 10 August 2020
}

five geographic regions of the country, but the latter is also registered in Suriname, French Guiana, Perú, Bolivia, Paraguay and northeast of Argentina. Whereas $N y$. neivai is restricted to colder and drier areas at Bolivia, Paraguay, North, West-Central, Southeast and South regions of Brazil, and north of Argentina. ${ }^{(2,3)}$ Another medical significant species is Lutzomyia longipalpis s.l. (Lutz \& Neiva), the most important neotropical vector of Leishmania infantum Nicolle, causative agent of visceral leishmaniasis. ${ }^{(5)}$ Its geographical distribution comprises a wide but discontinued range from central Mexico to Argentina and Uruguay, with a recently southern expansion and urban environments colonisation. ${ }^{(5,6)}$ A variety of evidence including chemical and behavioral suggests that it is a complex of at least seven species in Brazil, while molecular analyses identified eight haplogroups throughout its distribution. ${ }^{(5,7,8)}$ Regarding Lutzomyia cruzi (Mangabeira), its sibling, is also incriminated in Brazil as a vector of Le. infantum and suspected for transmitting Leishmania amazonensis Lainson \& Shaw. (9) While Lutzomyia alencari Martins, Souza \& Falcão, has not been incriminated as a vector of Leishmania species. ${ }^{(3)}$ The geographic distribution of $L u$. cruzi includes Bolivia and West-Central region of Brazil, while $L u$. alencari is restricted to southeast of Brazil. ${ }^{(2,3)}$

Closely-related species but reproductively isolated that appear morphologically identical are often referred 
to as cryptic or sibling species, and a group of cryptic species is referred to as a species complex. ${ }^{(10)}$ The existence of sibling or closely-related species can hamper the process of species determination. Inside Nyssomyia genus, both males and females of $N y$. intermedia, $N y$. whitmani and $N y$. neivai are closely-related species that can be determinate by subtle but sufficient morphological differences. ${ }^{(11)}$ On the other hand, inside Lutzomyia subgenus, the males of $\mathrm{Lu}$. longipalpis complex, Lu. alencari, Lu. cruzi, Lutzomyia pseudolongipalpis Arrivillaga \& Feliciangeli and Lutzomyia matiasi Le Pont \& Mollinedo presents very subtle details for their differentiation, while the female of $L u$. longipalpis complex, $L u$. cruzi and $L u$. matiasi are morphologically indistinguishable together with Lutzomyia gaminarai (Cordero, Vogelsang \& Cossio). ${ }^{(1)}$ Therefore, females of these species have been identified based on their site of collection and through their association with males collected from the same location. ${ }^{(1)}$ Molecular and biochemical findings suggest that reproductive barriers do not prevent gene flow among them, and evidence of introgression has been reported between $N y$. intermedia and $N y$. whitmani, and between $N y$. neivai and $N y$. intermedia. ${ }^{(12,13)}$ Similarly, Lu. longipalpis complex and $L u$. cruzi are not fully reproductively isolated with introgression occurring between them; the same is hypothesised for $L u$. longipalpis complex and $L u$. alencari in sympatric areas. ${ }^{(14,15,16)}$

Mitochondrial DNA (mtDNA) markers, as COI gene, have been used for phylogenetic relationships inference, phylogeography, population genetics and molecular taxonomic identification (DNA barcoding) in phlebotomine sand flies from the new and old world. ${ }^{(15,16,17,18,19)}$ Given the medical importance of some phlebotomine sand flies within Nyssomyia genus and Lutzomyia subgenus, the studies of phylogenetic relationships will increase our understanding of the evolutionary relationships within these closely-related species, which to date has not yet been explored. Gene flow between these phlebotomine sand flies may influence vector competence and capacity and could have important epidemiological consequences such as insecticide resistance or susceptibility to Leishmania infection. ${ }^{(13)}$ Therefore, the aim in the present study was to infer the phylogenetic relationships among closely-related phlebotomine sand flies within Nyssomyia genus ( $N y$. intermedia, Ny. whitmani and $N y$. neivai) and within Lutzomyia subgenus (Lu. longipalpis complex, $L u$. cruzi and Lu. alencari) using a COI fragment.

\section{MATERIALS AND METHODS}

Study area, collection and taxonomic identification - The Leishmaniasis Research Network in Argentina (REDILA) captured phlebotomine sand flies as part of different epidemiological projects in 11 localities from six Argentinean provinces: Corrientes, Entre Ríos, Formosa, Misiones, Salta and Tucumán during 2015-2018 [Fig. 1, Supplementary data (Table I)]. Phlebotomine sand flies captured using REDILA-BL ultraviolet light traps, were separated from other insects and were pre-

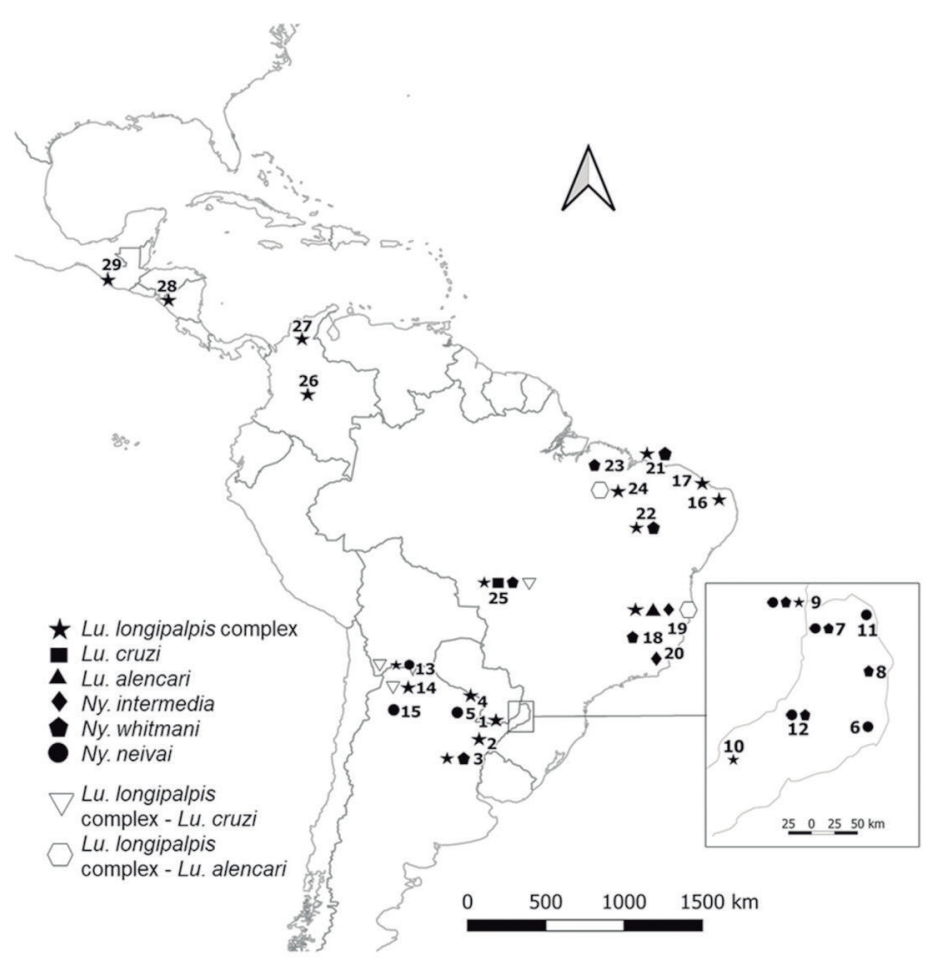

Fig. 1: geographic distribution of Lutzomyia longipalpis complex (stars), Lu. cruzi (square), Lu. alencari (triangle), Ny. intermedia (diamond), $N y$. whitmani (pentagon) and $N y$. neivai (circle) specimens analysed. Haplotypes shared: H5 and $\mathrm{H} 12$ between Lu. longipalpis complex and Lu. cruzi (white inverted triangle). H59 between Lu. longipalpis complex and Lu. alencari (white hexagon). For more information on numbers and species in the map, revise Supplementary data (Table I). 
served in $90 \%$ ethanol until being processed. For identification purposes, the head and the last three segments were dissected individually, clarified and mounted on slides while the rest of the body was stored at $-20^{\circ} \mathrm{C}$ until DNA extraction. Species determination was based on morphological characters according to Galati's taxonomic key, and genera abbreviations follow the nomenclature proposed by Marcondes..$^{(11,20)}$ Reference specimens have been deposited at the Instituto Nacional de Medicina Tropical, Puerto Iguazú, Argentina.

DNA extraction, amplification and sequencing - Genomic DNA was extracted individually from the rest of the body of the phlebotomine sand flies identified as $L u$. longipalpis complex, $N y$. neivai, $N y$. whitmani and one Migonemyia migonei (França). We used DNA PuriPrep$\mathrm{S}$ kit HighWay (INBIO, Argentina), following manufacturer's instruction, with the addition of an incubation period at $56^{\circ} \mathrm{C}$ that consisted in $4 \mathrm{~h}$ with vortex and spin every $20 \mathrm{~min}$. The $C O I$ fragment amplification was done by a polymerase chain reaction (PCR) using the universal primers. ${ }^{(21)}$ The amplification conditions were an initial denaturalisation step of $94^{\circ} \mathrm{C}$ for $4 \mathrm{~min}$, followed by 35 cycles of $94^{\circ} \mathrm{C}$ for $30 \mathrm{~s}, 56^{\circ} \mathrm{C}$ for $30 \mathrm{~s}, 72^{\circ} \mathrm{C}$ for 1 min, and a final extension of $72^{\circ} \mathrm{C}$ for $10 \mathrm{~min}$. PCR products were separated in $1.5 \%$ agarose gel electrophoresis, stained with $0.5 \mu \mathrm{L} / \mathrm{mL}$ SYBR Safe DNA (Invitrogen, USA) and isualized under blue light. The PCR products were purified with DNA PuriPrep-GP HW kit (INBIO, Argentina) following manufacturer's instruction. The sequencing was carried out using both forward and reverse primers on an ABI 3730XLs (Macrogen, Korea) or was sent to the Canadian Centre for DNA Barcoding.

Genetic analysis - Cytochrome $c$ oxidase subunit 1 (COI) sequences generated in this study of $N y$. whitmani, $N y$. neivai and Lu. longipalpis complex, (accession numbers in GenBank: MN857507-MN857540) in addition to sequences available from GenBank: Ny. intermedia (KP112715KP112761), Ny. whitmani (KP112762-KP112770; MG234392-MG234412), Lu. longipalpis complex (JN845539- JN845542; KP112581-KP112595; KT806402KT806405, KT806411, KT806413-KT806415, KT806417, KT806420, KT806426, KT806427, KT806430, KT806431, KT806437, KT806440, KT806443, KT806447, KT806451, KT806460, KT806468; GU909502-GU909505; HNLUZ004-HNLUZ005; MG234349-MG234370. BOLD Systems: MEXSM002-12.COI-5P, MEXSM003-12.COI5P), Lu. cruzi (KP112572-KP112577) and Lu. alencari (KP112569-KP112571) were included in the analysis [Supplementary data (Table I)]. All sequences were manually aligned and edited using MEGA v.10 software. The multiple alignment of all sequences, was carried out in MEGA v.10 software. The number of polymorphic sites by species, the number of haplotypes and nucleotide divergence $(D a)$ with the Jukes and Cantor (JC) correction among closelyrelated species were estimated using DnaSP v.6 software. Similarly, Kimura 2-parameter (K2P) genetic distances were estimated with 1000 replicates using MEGA v.10 software. The values of $D a$ and K2P between Lu. longipalpis complex clades (see results) were also estimated.
Haplotype network of closely-related species was constructed using Network v.4.6.1.3, with the Median Joining algorithm to evaluate the relationships among haplotypes shared and the frequency distribution among species. The best-fitting model of evolution was estimated using the Bayesian Information Criterion (BIC) as implemented in JModeltest v.0.1.1 software. Bayesian inference (BI) was analysed with MrBayes v.3.2 software, four Markov Chain Monte Carlo (MCMC) were run for 10 million generations (sampled every 1000 generations) to allow adequate time for convergence (all $\leq 0.004369$ ). The first $25 \%$ of sampled trees were considered as burned in. The tree was visualised using FigTree v.1.4 (http://tree.bio.ed.ac.uk/software/figtree/). Sequences of Mg. migonei (access number: MT430890) and Phlebotomus papatasi Scopoli (access number: KY848828) were used as outgroups.

\section{RESULTS}

Fifty-three phlebotomine sand flies were submitted to DNA extraction and COI amplification. Forty-eigth resulted in enough amplified product for sequencing ( 25 Nyssomyia genus and 23 Lutzomyia subgenus). Unfortunately, some of the sequences downloaded from GenBank and BOLD System were shorten than ours $(658$ bp). Then, during the multiple alignment all sequences were cut to the same length (543 bp for Nyssomyia genus and 483 bp for Lutzomyia subgenus).

Nyssomyia genus: $N y$. intermedia, $N y$. whitmani and $N y$. neivai - A total of $103 \mathrm{COI}$ sequences of $543 \mathrm{bp}$ were analysed: 47 sequences of $N y$. intermedia, 45 of $N y$. whitmani and 11 of $N y$. neivai. The species with more polymorphic sites was $N y$. whitmani (44 sites, $8.1 \%$ ). The values of K2P genetic distance ranged from 3.1 to $3.5 \%$, while, the nucleotide divergence $(D a)$ ranged from 0.017 to 0.025 . The maximum and minimun K2P and $D a$ values were between $N y$. intermedia vs $N y$. neivai and $N y$. whitmani vs $N y$. neivai, respectively (Table I).

Seventy-one haplotypes were identified from 103 Nyssomyia sequences, none haplotype was shared among them. Twenty-two haplotypes were identified in $N y$. intermedia from localities of Brazil, 38 in $N y$. whitmani from localities of Brazil and Argentina and 11 in $N y$. neivai from Argentinean localities. The medianjoining network identified at least 11 mutational steps between $N y$. intermedia vs $N y$. neivai and $N y$. whitmani vs $N y$. neivai, while between $N y$ intermedia vs $N y$. whitmani there were at least 22 mutational steps. Argentinean Ny. whitmani (H61) from the northeast (Gobernador Lanusse and Piñalito Sur, Misiones provinces) and Pampeana (Concordia, Entre Ríos province) regions were identified by at least three mutational steps from Brazilian specimens (H58, Maranhão, Mato Grosso and Espírito Santo states) (Fig. 2). Bayesian inference (BI) was constructed using the $\mathrm{HKY}+\mathrm{I}+\mathrm{G}$ model as the most appropriate for the data $(-\operatorname{lnL}=2152.4292 ; \mathrm{BIC}=$ 5243.1277; Delta $\mathrm{BIC}=0$ ) with gamma of 0.1080 and $\mathrm{p}$-inv of 0.6680 . The analysis provided support with 0.77 posterior probability (PP) for the clade of $N y$. intermedia and Ny. whitmani. Nyssomyia intermedia specimens 


\section{TABLE I}

Kimura 2-parameter (K2P) pairwise genetic distances between closely-related phlebotomine species within the Nyssomyia genus (below diagonal) and nucleotide divergence $(D a)$ of the cytochrome $c$ oxidase subunit I $(C O I)$ fragment measured as the number of net nucleotide substitution (above diagonal)

\begin{tabular}{llll}
\hline & Ny. intermedia & Ny. whitmani & Ny. neivai \\
\cline { 2 - 3 } Ny. intermedia & & $0.021 \pm 0.001$ & $0.025 \pm 0.003$ \\
\hline Ny. whitmani & $0.034 \pm 0.006$ & & $0.017 \pm 0.002$ \\
\hline Ny. neivai & $0.035 \pm 0.007$ & $0.031 \pm 0.006$ & \\
\hline
\end{tabular}

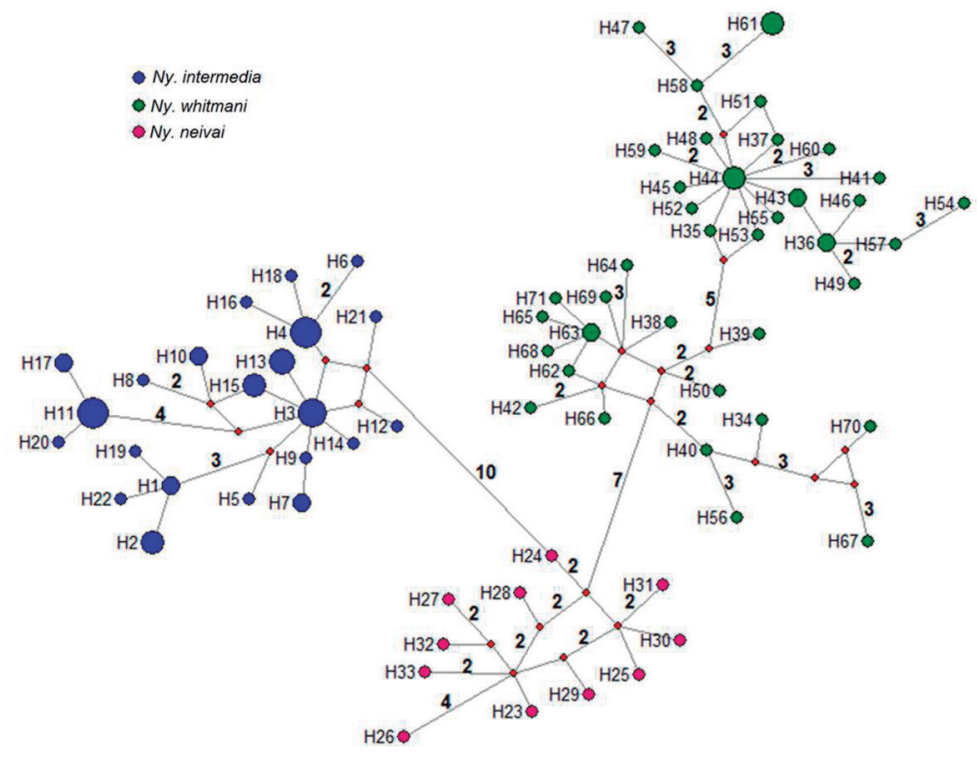

Fig. 2: Median-joining haplotype network for Nyssomyia intermedia, $N y$. whitmani and Ny. neivai based on 543 nucleotides of the cytochrome $c$ oxidase subunit I (COI) gene. Haplotype frequency is represented by the size of nodes and missing haplotypes are shown as red circles. The line connecting haplotypes represents one mutational step, whereas numbers along the lines are total number of mutational steps. Colours indicate: blue $=N y$. intermedia , green $=N y$. whitmani; pink $=N y$. neivai .

grouped monophyletically with $0.96 \mathrm{PP}$, while some $N y$. whitmani specimens also grouped monophyletically with $0.92 \mathrm{PP}$ and another group presented polytomies (a comb-shaped phylogeny). Likewise, the basal clade with Ny. neivai specimens presented polytomies (Fig. 3).

Lutzomyia subgenus: Lu. longipalpis complex, Lu. cruzi and Lu. alencari - A total of 103 sequences of a 483 bp COI fragment were analysed: 94 sequences of Lu. longipalpis complex, six of Lu. cruzi and three $L u$. alencari. Unfortunately, there were no sequences available in GenBank of Lu. pseudolongipalpis, Lu. matiasi and $L u$. gaminarai, which are also closely-related species within the Lutzomyia subgenus.

Lutzomyia longipalpis complex showed the highest number of polymorphic sites (107 sites, 22\%). The K2P genetic distance (ranged from 2.5 to $3.4 \%$ ) and the nucleotide divergence $(D a)$ (from 0.005 to 0.016 ) between closely-related species were low. The maximum and minimun K2P values were between $L u$. longipalpis complex vs $L u$. cruzi and Lu. longipalpis complex vs Lu. alencari, respectively. In contrast, the maximum and minimun $D a$ values were between $L u$. cruzi vs $L u$. alencari and Lu. longipalpis complex vs Lu. cruzi and Lu. alencari (Table II). The intraspecific K2P distance and $D a$ between clades from $\mathrm{Lu}$. longipalpis complex (1 vs 2) (K2P = 9.8\%, Da $=0.08$ ) were high. Likewise, the values between clade 2 (samples from Colosó, Sucre) vs $\mathrm{Lu}$. cruzi (K2P $=9.8 \%$, $D a=0.081)$ and vs Lu. alencari $(\mathrm{K} 2 \mathrm{P}=9.6 \%, D a=0.089)$ also were high [Supplementary data (Table II)].

Seventy-eight haplotypes were identified from 103 sequences analysed of the three closely-related species of Lutzomyia subgenus: 72 haplotypes of Lu. longipalpis complex, six of Lu. cruzi and three of Lu. alencari. The minimum spanning haplotype network showed three haplotypes shared among species: H5 was shared between Lu. longipalpis complex (Mato Grosso, Brazil and Tartagal, Argentina) and Lu. cruzi (Mato Grosso, Brazil); H12 was shared between Lu. longipalpis com- 


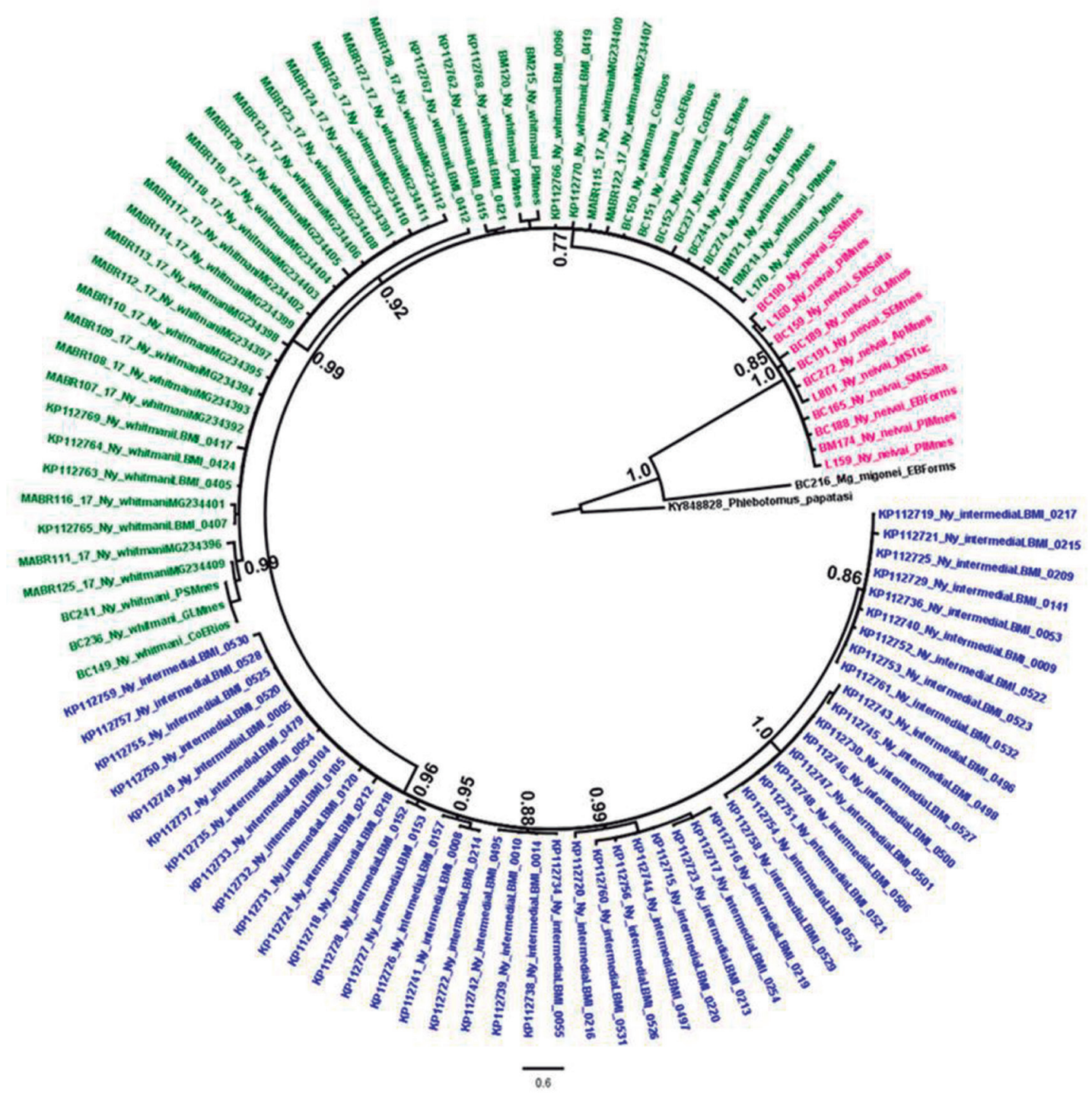

Fig. 3: Bayesian inference (BI) topology tree for 543 nucleotides of the cytochrome $c$ oxidase subunit I (COI) gene of Nyssomyia intermedia, $N y$. whitmani and $N y$. neivai inferred using the $\mathrm{HKY}+\mathrm{I}+\mathrm{G}$ model. Numbers on each Branch (above branch) represent posterior probabilities (PP) obtained in the BI. Migonemyia migonei and Phlebotomus papatasi were used as outgroup. The scale bar represents the expected number of nucleotide. Colours indicate: blue $=N y$. intermedia , green $=N y$. whitmani; pink $=N y$. neivai.

plex and $\mathrm{Lu}$. cruzi (both from Mato Grosso, Brazil); and H59 was shared between Lu. longipalpis complex (Maranhão, Brazil) and Lu. alencari (Espírito Santo, Brazil). The specimens of $\mathrm{Lu}$. longipalpis complex from Salta H6-H7, Tartagal H69 (Argentina) and Cáceres H11 (Brazil) are at least at one mutational step of $L u$. cruzi H73 and H75 from Cáceres, Mato Grosso (Brazil). On the other hand, Lu. longipalpis complex specimens from Chiapas H71-H72 (Mexico) and Valle H26-H27 (Honduras) are separated by at least 24 and 26 mutational steps from Cundinamarca H28-H31 (Colombia, Andean region) and São Domingos do Maranhão H56 (Brazil) specimens, respectively (Fig. 4). Bayesian inference was constructed using the $\mathrm{HKY}+\mathrm{G}$ model as the most appropriate for the data $(-\operatorname{lnL}=2271.2317 ; \mathrm{BIC}=$ 5852.6268; Delta $\mathrm{BIC}=0$ ) with gamma of 0.1040 . The tree showed similar results to the network, its topology did not present structuration by species either by geography. Two monophyletic clades were identified: the first grouped specimens of $\mathrm{Lu}$. longipalpis complex, $\mathrm{Lu}$. cruzi and Lu. alencari (1.0 PP), while the second clade grouped only $L u$. longipalpis complex specimens from Colosó, Colombia with 1.0 PP (Caribbean region) (Fig. 5). Indeed, Lu. longipalpis complex specimens from Colombia (Ricaurte, Cundinamarca and Colosó, Sucre) grouped within two monophyletic separated clades, the haplotype network identified at least 68 mutational steps between them (Figs 4-5). 
TABLE II

Kimura 2-parameter (K2P) pairwise genetic distances between closely-related phlebotomine species within Lutzomyia subgenus (below diagonal) and nucleotide divergence $(D a)$ of the cytochrome $c$ oxidase subunit I $(C O I)$ fragment measured as the number of net nucleotide substitution (above diagonal)

\begin{tabular}{llll}
\hline & Lu. longipalpis complex & Lu. cruzi & Lu. alencari \\
\cline { 2 - 4 } Lu. longipalpis complex & & $0.005 \pm 0.003$ & $0.005 \pm 0.003$ \\
\hline Lu. cruzi & $0.034 \pm 0.005$ & & $0.016 \pm 0.009$ \\
\hline Lu. alencari & $0.025 \pm 0.004$ & $0.030 \pm 0.006$ & \\
\hline
\end{tabular}

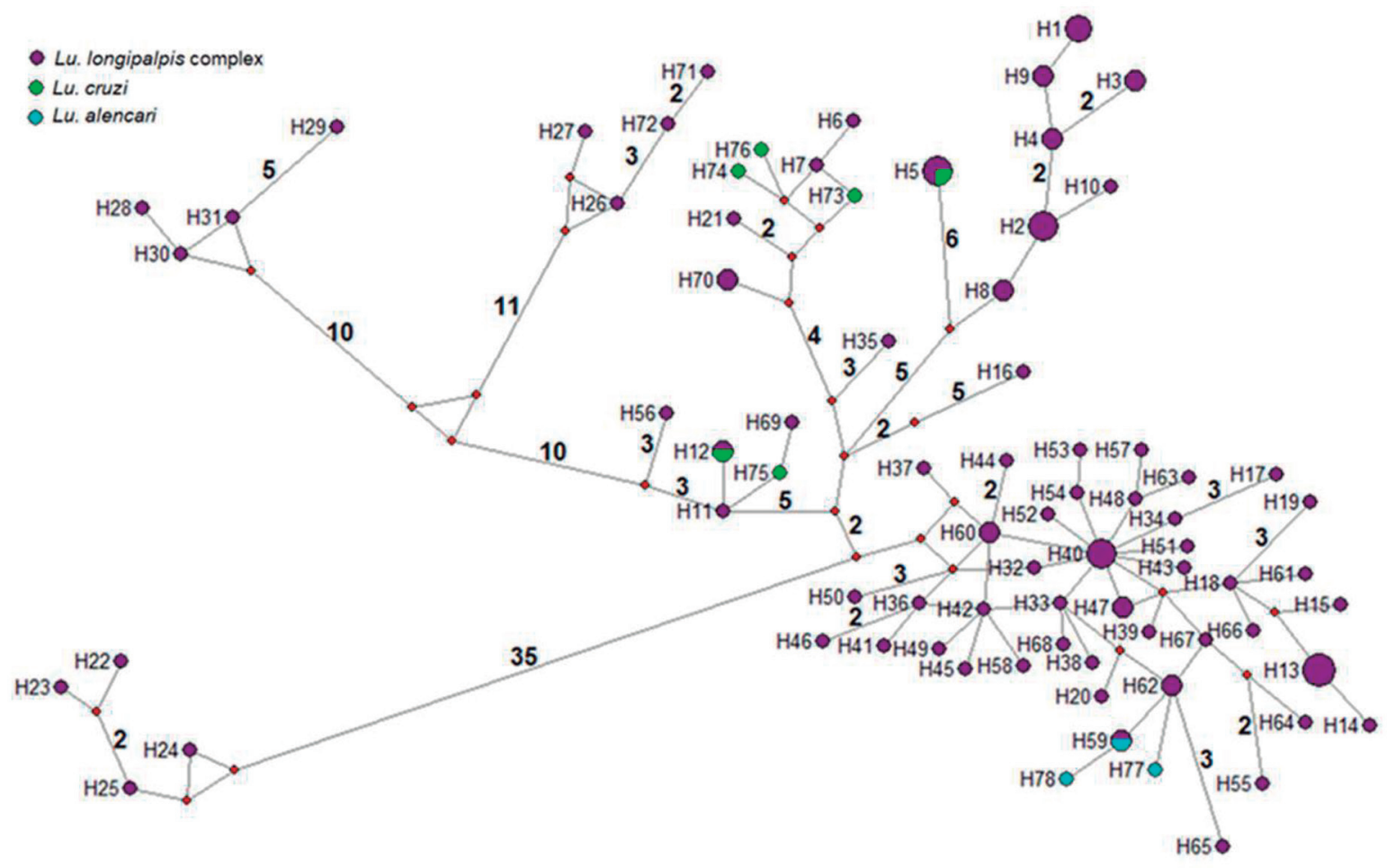

Fig. 4: Median-joining haplotype network for the Lutzomyia longipalpis complex, Lu. cruzi and Lu. alencari based on 483 nucleotides of the cytochrome $c$ oxidase subunit I (COI) gene. Haplotype frequency is represented by the size of nodes and missing haplotypes are shown as red circles. The line connecting haplotypes represents one mutational step, whereas numbers along the lines are total number of mutational steps. Colours indicate: purple $=L u$. longipalpis complex; green $=L u$. cruzi; turquoise $=L u$. alencari .

\section{DISCUSSION}

The findings of the present study evidence low values of both K2P genetic distance and nucleotide divergence (Da) between closely-related species within Nyssomyia genus and within Lutzomyia subgenus. The genetically closest species within Nyssomya genus were Ny. whitmani and Ny. neivai; while within Lutzomyia subgenus were Lu. longipalpis complex with Lu. alencari and with Lu. cruzi. Inside Nyssomyia genus, Mazzoni et al. ${ }^{(12,22)}$ reported lower divergence values than us between $N y$. intermedia and Ny. whitmani from southeast Brazil using ten nuclear loci. This can be due to: (a) Ny. neivai specimens were not analysed, (b) differences in nucleotide substitution rate between nuclear and mitochondrial genes, (c) the number of samples analysed, (d) the small- er geographical area they assessed or (e) a combination among them. Currently, de Melo et al. ${ }^{(23)}$ reported even lower interspecific values inside Bichromomyia flaviscutellata complex between Bichromomyia olmeca olmeca (Vargas and Díaz-Nájera) and B. olmeca bicolor (Fairchild and Theodor). These authors concluded that these closely-related species represent the same species, supporting the morphological data. On the other hand, Scarpassa and Alencar, ${ }^{(18)}$ reported higher interspecific values (4.1-4.6\%) between Nyssomyia anduzei (Rozeboom) and Nyssomyia umbratilis (Ward \& Fraiha). Both are closely-related species with extensive overlapping areas and high morphological similarity. Arrivillaga et al. ${ }^{(17)}$ reported higher values of $\mathrm{K} 2 \mathrm{P}$ distance ranging 1.3-17.7\% within Lu. longipalpis complex while between 


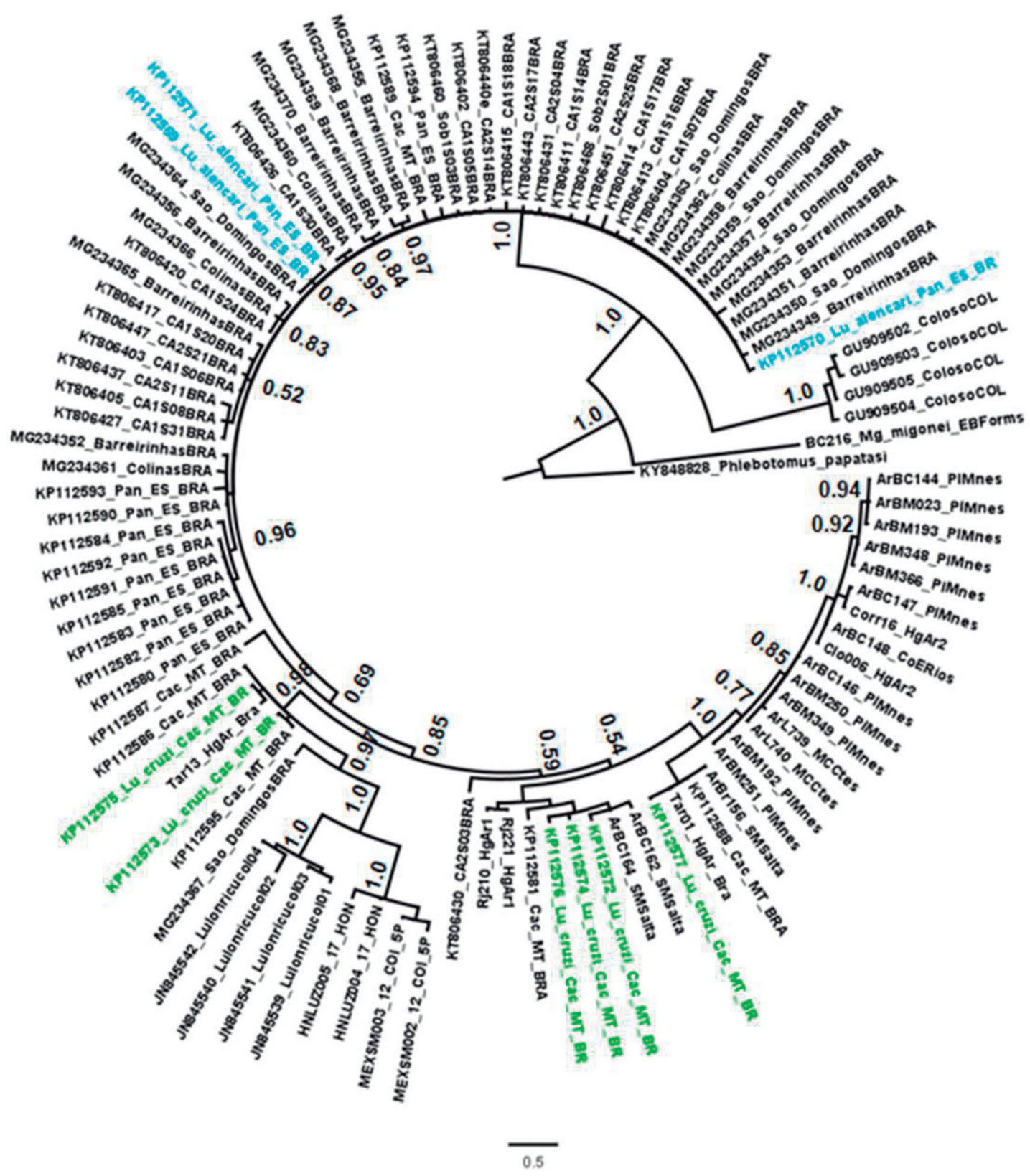

Fig. 5: Bayesian inference (BI) topology tree for 483 nucleotides of the cytochrome $c$ oxidase subunit I (COI) gene of Lutzomyia longipalpis complex, Lu. cruzi and Lu. alencari inferred using the HKY + G model. Numbers on each Branch (above branch) represent posterior probabilities (PP) obtained in the BI. Migonemyia migonei and Phlebotomus papatasi were used as outgroup. The scale bar represents the expected number of nucleotides. Colors indicate: black $=L u$. longipalpis complex; green $=L u$. cruzi; turquoise $=L u$. alencari .

Lu. longipalpis haplogroups (Hg) were 10.1-13\%. In addition, K2P distance between Colombians specimens was comparable with the distance found between $L u$. longipalpis complex clades (1 vs 2 ) in the present study. This could be explained by the geographical barriers in Colombia, such as the Andean Cordillera. ${ }^{(8,17)}$

The haplotype network and BI of Nyssomyia genus showed structuring by species although presented some polytomies, probably due to lack of samples. None haplotype was shared among species, contrary to mitochondrial introgression reported by Marcondes et al. ${ }^{(13)}$ between $N y$. intermedia and $N y$. whitmani as well as between $N y$. intermedia and $N y$. neivai using cyt $b$ gene. Moreover, nuclear introgression using period gene was reported between $N y$. intermedia and $N y$. whitmani from southeast Brazil. ${ }^{(12,22)}$ In the present study, haplotype network separated these three closely-related species by various mutational steps. Indeed, $N y$. intermedia and $N y$. whitmani have 22 mutational steps between them. All $N y$. intermedia specimens from Espírito Santo (Brazil) presented few mutational steps among haplotypes, may be due to the restricted geographical range covered. 
Similarly, Meneses et al. ${ }^{(24)}$ reported in Ny. intermedia population from Rio Janeiro (Brazil) few intra-population level polymorphism, using COI, $12 \mathrm{~S}$ and $16 \mathrm{~S}$. In the BI, $N y$. whitmani presented one monophyletic group (0.92 PP) and the rest of the specimens did not group (presenting polytomies). These not-grouped specimens from different localities of Brazil and Argentina are at least at seven mutational steps from the monophyletic group; suggesting that may form another monophyletic clade although more samples are needed to confirm this hypothesis. Indeed, haplotype network showed missing intermediate haplotypes between the two $N y$. whitmani groups. Interestingly, Argentinian Ny. whitmani haplotypes from Concordia (its southernmost distribution until now) $\mathrm{H} 61$ and H62/H63 are separated by at least 17 mutational steps. ${ }^{(6)}$ Similarly, Ny. whitmani haplotypes from Puerto Iguazú (Northeast region, Argentina) have at least 11 mutational steps between them. These several mutational steps between the Ny. whitmani haplotypes from Argentina suggest that it could be a complex species occurring in sympatry. Ready et al. ${ }^{(4)}$ using cyt $b$ gene suggested the presence of at least three phylogenetic lineages of Ny. whitmani in Brazil. Ishikawa et al. ${ }^{(25)}$ adding samples of $N y$. whitmani from the State of Rondônia (Brazil), identified two clades, one from eastern Amazônia and one from the Atlantic forest zone of the northeast, although with low support value $(<50.0 \%$ bootstrap). Unfortunately, in the present study, $N y$. whitmani specimens did not cover the same locations previously studied by Ready et al. ${ }^{(4)}$ and Ishikama et al. ${ }^{(25)}$ to compare the lineages. Regarding Ny. neivai, there are no studies available for comparisons, being these $\mathrm{COI}$ sequences the first available in GenBank.

Lutzomyia subgenus haplotype network and BI showed topologies without structuration by species neither by geography. The genetically closest species were $L u$. longipalpis complex with $L u$. alencari and with $L u$. cruzi. Lutzomyia longipalpis complex shared haplotypes with both species or was found at least at one mutational step of them, compared with $L u$. cruzi vs Lu. alencari that are separated by at least 16 mutational steps. Three haplotypes were shared among these species: (1) H5, Lu. longipalpis complex specimens from Argentina (Ar-Bra $\mathrm{Hg}$ ) with $L u$. longipalpis complex and $L u$. cruzi from central-western Brazil, (2) H12, Lu. longipalpis complex with $L u$. cruzi (both from central-western Brazil) and (3) H59, Lu. longipalpis complex (northeast Brazil) with $\mathrm{Lu}$. alencari (southeast Brazil). In this sense, the shared haplotypes detected both in symphatry and allophatrycally may be explained by introgression among these species. The introgressive hybridisation seems to be a recurrent phenomenon that shaped the genome of Lu. longipalpis complex. ${ }^{(26)}$ This was suggested for $L u$. longipalpis complex and $L u$. cruzi based on molecular analyses using microsatellites markers ${ }^{(14)}$ and morphometrics analyses in sympatric areas. ${ }^{(27)}$

In addition, it was reported that the pheromone chemotype 1 (9MGB) was found in $L u$. cruzi specimens (Bolivia and Brazil) and some Lu. longipalpis complex specimens from Honduras, Venezuela, Colombia, Brazil, Paraguay and Argentina. ${ }^{(7,28)}$ Studies carried out by
Pinto et al..$^{(15)}$ and Rodrigues et al. ${ }^{(16)}$ on molecular taxonomic identification, supported the possibility of introgression events between Lu. longipalpis complex and both $L u$. cruzi and $L u$. alencari. In neighbour-joining analysis, they reported that Lu. longipalpis complex formed two groups, one included $L u$. cruzi specimens from the Central-Western region, and the other grouped with $L u$. alencari from Southeastern region (Brazil); however, they did not mention the existence of haplotypes shared among these closely-related species. Pinto et al. ${ }^{(15)}$ suggested that the clustering pattern is associated with the geographic distribution of $L u$. cruzi and $L u$. alencari and their sympatry with $\mathrm{Lu}$. longipalpis complex..$^{(3)}$ However, we detected two shared haplotypes approximately $1000 \mathrm{~km}$ apart (H5; H59). Concerning $L u$. longipalpis complex itself, we included samples of the three genetic differentiated haplogroups (Ar1, Ar2, ArBra) described in Argentina using ND4 and cyt $b$ genes. ${ }^{(8)}$ We suggest that Ar2 could be the haplogroup that is spreading its distribution to southern Argentina given that Concordia's specimen of Lu. longipalpis complex shared the H4 with Clorinda's specimens. Concordia is located $800 \mathrm{~km}$ south of Clorinda and both localities belong to different ecoregions (Spinal and Humid Chaco, respectively). Furthermore, in BI Concordia's specimen grouped with another specimen of Ar2 haplogroup also previously identified (1.0 PP). To test this hypothesis, more specimens and other genetic markers are needed.

The second monophyletic clade of Lutzomyia subgenus is represented only by Colombian specimens of $L u$. longipalpis complex from Colosó, Sucre. The others Colombian specimens from Ricaurte, Cundinamarca clustered within clade 1, closer to specimens from Honduras and Mexico. Arrivillaga et al. ${ }^{(17)}$ reported specimens from Bucaramanga within the trans-Andean haplogroup and specimens from Bucaramanga and Neiva within the cis-Andean haplogroup using another $\mathrm{COI}$ gene fragment. Similarly, Pech-May et al. ${ }^{(8)}$ using ND4 and cyt $b$ genes also found divergence in Lu. longipalpis complex from Colombia: Giron (Coll Hg) vs El Callejón and Neiva $(\mathrm{Col} 2 \mathrm{Hg})$ with at least 71 mutational steps between them, and a high genetic differentiation value $\left(\mathrm{F}_{\mathrm{ST}}=0.966\right)$ suggesting negligible gene exchange. In this study, the minimum spanning haplotype network showed that Colombian clades of $\mathrm{Lu}$. longipalpis complex are separated by at least 68 mutational steps. This divergence in Lu. longipalpis complex from Colombia could be potentially due to vicariance, geographic and/or climatic barriers in addition to its low dispersal capacity. ${ }^{(8,17)}$ Contrary, the few genetic distance detected among closely-related species is probably related to evolutionary factors like incipient speciation and genetic introgression. ${ }^{(19)}$ The last one, as a consequence of natural hybridisation, might make the species to share traits of biomedical importance like vector competence, insecticide resistance, behavioral phenotypes and environmental adaptability, highlighting the need to focus on gene flow and the distribution of phenotypes of biomedical importance. ${ }^{(29)}$ Pinto et al. ${ }^{(30)}$ suggested that the phylogenetic proximity among Lutzomyia subgenus is congruent with their role as vectors of the same parasite; given that the physiologic mechanisms of these insects are likely 
to be similar due to recent common ancestry. Although Lu. alencari, Lu. pseudolongipalpis and Lu. matiasi have not been found naturally infected with Leishmania, attention should be given to their potential role in Leishmania transmission cycles, as these species are sister to incriminated vectors ( $\mathrm{Lu}$. longipalpis complex and $\mathrm{Lu}$. cruzi).

This is the first study that evidence the phylogenetic proximity among closely-related phlebotomine species within Nyssomyia genus and within Lutzomyia subgenus. The relationships described here should be assessed using additional molecular markers (mitochondrial, nuclear, SNPs or microsatellites) with a broad sampling of all closely-related species, including to $\mathrm{Lu}$. pseudolongipalpis, Lu. gaminarai, Lu. matiasi. In the case of $L u$. longipalpis complex could be interesting to include all the haplogroups, given that the genetic distance seems to be higher within the complex that among the closely-related species. The presented results can be used as testable phylogenetic hypotheses and as a phylogenetic framework to future research using an integrative approach combining morphological, behavioral, chemical and multiple molecular markers with different evolution rate. Knowledge of phylogenetic relationships among relevant visceral and cutaneous leishmaniasis vectors and its siblings species (especially in sympatric areas) could be useful also in epidemiology control and strategies design, helping to assess if other species could be suspected of having a vectorial role in endemic leishmaniases areas.

\section{ACKNOWLEDGEMENTS}

To Elizabet Vilacoba and Dario Lijtmaer from the Museo Argentino de Ciencias Naturales Bernardino Rivadavia. We are also grateful to Javier Liotta, Magali Giuliani for their assistance in the laboratory; Daniela Lamattina for providing samples (Instituto Nacional de Medicina Tropical - ANLIS Dr Carlos G Malbrán), and the workgroup of Concordia municipality.

\section{AUTHORS' CONTRIBUTION}

Conceptualisation - AP-M and SLM; formal analysis AP-M; funding acquisition - ODS, SLM, MM-A and MGQ; methodology - AP-M, SLM, MGQ and MM-A; writing/ original draft - AP-M and SLM; writing/review and editing AP-M, SLM, MGQ, MM-A and ODS.

\section{REFERENCES}

1. Ready PD. Biology of phlebotomine sand flies as vectors of disease agents. Annu Rev Entomol. 2013; 58: 227-50.

2. Shimabukuro PHF, De Andrade AJ, Galati EAB. Checklist of American sand flies (Diptera, Psychodidae, Phlebotominae): genera, species, and their distribution. Zookeys. 2017; 660: 67-106.

3. Aguiar GM, Vieira VR. Regional distribution and habitats of Brazilian phlebotomine species. In: Rangel EF, Shaw JJ, editors. Brazilian sand flies. Switzerland: Springer; 2018. p. 251-98.

4. Ready PD, Day JC, de Souza AA, Rangel EF, Davies CR. Mitochondrial DNA characterization of populations of Lutzomyia whitmani (Diptera: Psychodidae) incriminated in the peri-domestic and silvatic transmission of Leishmania species in Brazil. Bull Entomol Res. 1997; 87(2): 187-95.

5. de Souza NA, Brazil RP, Araki AS. The current status of the $L u$ tzomyia longipalpis (Diptera: Psychodidae: Phlebotominae) species complex. Mem Inst Oswaldo Cruz. 2017; 112(3): 161-74.
6. Santini MS, Manteca Acosta M, Utgés ME, Aldaz ME, Salomón OD. Presence of Lutzomyia longipalpis and Nyssomyia whitmani in Entre Rios, Argentina. Rev Inst Med Trop São Paulo. 2018; 60: e55.

7. Araki AS, Vigoder FM, Bauzer LGSR, Ferreira GEM, Souza NA, Araújo IB. Molecular and behavioral differentiation among Brazilian populations of Lutzomyia longipalpis (Diptera: Psychodidae: Phlebotominae). PLoS Negl Trop Dis. 2009; 3(1): e365.

8. Pech-May A, Ramsey J, González Ittig R, Giuliani M, Berrozpe $\mathrm{P}$, Quintana $\mathrm{M}$, et al. Genetic diversity, phylogeography and molecular clock of the Lutzomyia longipalpis complex (Diptera: Psychodidae). PLoS Negl Trop Dis. 2018; 12(7): e0006614.

9. Falcão de Oliveira E, Oshiro ET, Fernandes WS, Ferreira AMT, Gutierrez de Oliveira A, Galati EAB. Vector competence of $L u$ tzomyia cruzi naturally demonstrated for Leishmania infantum and suspected for Leishmania amazonensis. Am J Trop Med Hyg. 2017; 96(1): 178-81.

10. Black WC, Munstermann LE. Molecular taxonomy and systematics of arthropods vectors. In: Marquardt WC, editor. Biology of disease vectors. Ámsterdam: Elsevier Academic Press; 2004. 505-28.

11. Galati EAB. Phebotominae (Diptera: Psychodidae): clasification, morphology and terminology of adults and identification of American taxa. Morfologia e terminologia de Phlebotominae (Diptera: Psychodidae). In: Rangel EF, Shaw JJ, editors. Brazilian sand flies. Switzerland: Springer. 2018; 9-212.

12. Mazzoni CJ, Araki AS, Ferreira GEM, Azevedo RVDM, Barbujani G, Peixoto AA. Multilocus analysis of introgression between two sand fly vectors of leishmaniasis. BMC Evol Biol. 2008; 8: 141.

13. Marcondes CB, Day JC, Ready PD. Introgression between Lutzomyia intermedia and both $L u$. neivai and Lu. whitmani, and their roles as vectors of Leishmania braziliensis. Trans R Soc Trop Med Hyg. 1997; 91(6): 725-6.

14. Santos MFC, Ribolla PEM, Alonso DP, Andrade-Filho JD, Casaril AE, Ferreira AMT, et al. Genetic structure of Lutzomyia longipalpis populations in Mato Grosso do Sul, Brazil, based on microsatellite Markers. PLoS One. 2013; 8: e74268.

15. Pinto I, Chagas BD, Rodrigues AAF, Ferreira AL, Rezende HR, Bruno RV, et al. DNA barcoding of neotropical sand flies (Diptera, Psychodidae, Phlebotominae): species identification and discovery within Brazil. PLoS One. 2015; 10(10): e0140636.

16. Rodrigues BL, Carvalho-costa LF, Pinto IS, Manuel J, Rebêlo M. DNA barcoding reveals hidden diversity of sand flies (Diptera: Psychodidae) at fine and broad spatial scales in Brazilian endemic regions for leishmaniasis. J Med Entomol. 2018; 55(4): 893-901.

17. Arrivillaga JC, Norris DE, Feliciangeli MD, Lanzaro GC. Phylogeography of the neotropical sand fly Lutzomyia longipalpis inferred from mitochondrial DNA sequences. Infect Genet Evol. 2002; 2: 83-95.

18. Scarpassa VM, Alencar RB. Molecular taxonomy of the two Leishmania vectors Lutzomyia umbratilis and Lutzomyia anduzei (Diptera: Psychodidae) from the Brazilian Amazon. Parasit Vectors. 2013; 6: 258.

19. Freitas M, Costa Jr C, Sillva L, Leal-Balbino T, Balbino V. Cytochrome oxidase I as tool to evaluate the Lutzomyia longipalpis complex: useful molecular marker or not? Vector Biol J. 2016; 1(3): 1-4.

20. Marcondes CB. A proposal of generic and subgeneric abbreviations for phlebotomine sandflies (Diptera: Psychodidae: Phlebotominae) of the world. Entomol News. 2007; 118(4): 351-6.

21. Folmer O, Black M, Hoeh W, Lutz R, Vrijenhoek R. DNA primers for amplification of mitochondrial cytochrome $c$ oxidase subunit I from diverse metazoan invertebrates. Mol Mar Biol Biotechnol. 1994; 3(5): 294-9. 
22. Mazzoni CJ, Souza NA, Andrade-Coelho C, Kyriacou CP, Peixoto AA. Molecular polymorphism, differentiation and introgression in the period gene between Lutzomyia intermedia and Lutzomyia whitmani. BMC Evol Biol. 2006; 6: 85.

23. de Melo LB, Alencar RB, Scarpassa VM. Molecular taxonomy and phylogenetic inferences of Bichromomyia flaviscutellata complex based on the COI gene DNA barcode region. Infect Genet Evol. 2020; 81: 104256.

24. Meneses C, Cupolillo E, Monteiro F, Rangel E. Micro-geographical variation among male populations of the sandfly, Lutzomyia (Nyssomyia) intermedia, from an endemic area of American cutaneous leishmaniasis in the State of Rio de Janeiro, Brazil. Med Vet Entomol. 2005; 19(1): 38-47.

25. Ishikawa EAY, Ready PD, de Souza AA, Day JC, Rangel EF, Davies $\mathrm{CR}$, et al. Mitochondrial DNA phylogeny indicates close relationships between populations of Lutzomyia whitmani (Diptera: Psychodidae, Phlebotominae) from the Rain-forest Regions of Amazônia and Northeast Brazil. Mem Inst Oswaldo Cruz. 1999; 94(3): 339-45.
26. Araki AS, Ferreira GEM, Mazzoni CJ, Souza NA, Machado RC, Bruno RV, et al. Multilocus analysis of divergence and introgression in sympatric and allopatric sibling species of the Lutzomyia longipalpis complex in Brazil. PLoS Negl Trop Dis. 2013; 7: e2495.

27. Santos MFC, Andrade-Filho JD, Fernandes CES, Mateus NLF, Eguchi GU, Fernandes WD, et al. Morphometric analysis of Longipalpis (Diptera: Psychodidae) complex populations in Mato Grosso do Sul, Brazil. J Med Entomol. 2015; 52(3): 359-67.

28. Spiegel CN, Dias DB, Araki AS, Hamilton JGC, Brazil RP, Jones TM. The Lutzomyia longipalpis complex: a brief natural history of aggregation-sex pheromone communication. Parasit Vectors. 2016; 9(1): 1-15.

29. Ready PD. Should sand fly taxonomy predict vectorial and ecological traits? J Vector Ecol. 2011; 36(Suppl. 1): S17-22.

30. Pinto I, Andrade Filho J, Santos C, Falqueto A, Leite Y. Phylogenetic relationships among species of Lutzomyia, subgenus Lutzomyia (Diptera: Psychodidae). J Med Entomol. 2010; 47(1): 16-21. 